\title{
ANALYSIS OF THE DEGREE OF ADHERENCE TO THERAPY IN PATIENTS WITH DIAGNOSED DEPRESSION
}

\section{Antonia Kondova ${ }^{1}$, Anna Todorova ${ }^{2}$, Antoaneta Tsvetkova ${ }^{3}$, Kalina Andreevska ${ }^{4}$, Daniela Grekova $^{5}$, Valentina Petkova ${ }^{6}$}

\begin{abstract}
:
Introduction: Depression is the most common form of mental disorder of great social significance. Antidepressant treatment is a standard approach to treat depressed patients, but in order to be effective, patients need to follow their strictly prescribed treatments. Bad compliances are mostly associated with side effects, poor patient awareness, comorbidity of the disease, and a lack of communication with healthcare professionals.

Objectives: The aim is to investigate the level of adherence to the therapy of patients with a diagnosed depressive condition.

Methods: In order to achieve this aim, a survey was conducted among 120 patients with diagnosed depression when receiving their prescribed drugs from a pharmacy. Determination of patient's adherence was done using the Morriski test- as well as a short questionnaire in parallel as a validated tool giving a clear idea of the degree of adherence to therapy.

Results: The results show that the patients under study exhibited unsatisfactory adherence to the assigned therapy. The total coefficient for the entire population is 1.68 . Compared with the reference values (0-4), patients were found to have $42 \%$ adherence.

Conclusions: Inadequate adherence to therapy leads to a worsening of the condition of patients with depression while also increasing the cost of health care. A number of approaches are required such as patient education, quality communication between doctors, patients and pharmacists, socio-economic measures to change patient's beliefs and perceptions of treatment, and to improve adherence to the therapy and quality of life of patients suffering from depression.
\end{abstract}

UDC Classification: 615.1; DOI: http://dx.doi.org/10.12955/cbup.v6.1271

Keywords: depression, adherence, Morriski test

\section{Introduction:}

Depression is the most common form of mental disorder, which is of great importance to society. The World Health Organization puts depression among the most debilitating diseases in the world, with severe depression being placed in a disability category with the end-stage malignant neoplasms. Antidepressant treatment is a standard approach to treat depressed patients, but to be effective, patients need to follow their strictly prescribed treatment. Failure to receive therapies has the extent of an epidemic in recent years. Bad compliance is mostly associated with side-effects, poor patient awareness, comorbidity of the disease, and a lack of communication with healthcare professionals.

\section{Literature review:}

Depression is a disease known for millennia but continues to affect more people in the world and in our country. Depression is considered as a socially significant disease, especially in more developed countries. Depression can affect anyone, and it is one of the most widespread illnesses, often coexisting with other serious illnesses (World Federation for Mental Health Day, 2012).

Non-adherence to one's therapy is a leading factor in failure to treat depression worldwide. This is a major problem, especially with patients suffering from more than one disease. Therapeutic adherence can be influenced by a number of factors and the some of the most important ones are the patient's desire to take care of themselves, their knowledge of the disease and the risks it hides, their perceptions of the benefits of treatment and a number of other factors. Therapeutic consent plays a key role in achieving clinical outcomes. Adherence to therapy is a complicated process where the patient with their complex human behavior, their close relatives and professionals are sharing the responsibility. To achieve clinical outcomes in patients with chronic illness, it is essential that patients agree with the therapy and their attitude towards the prescribed regimen.

\footnotetext{
${ }^{1}$ Faculty of Pharmacy, Medical University of Varna, Bulgaria, antoniakondova@ mail.bg

${ }^{2}$ Faculty of Pharmacy, Medical University of Varna, Bulgaria, annitodorova@abv.bg

${ }^{3}$ Education and Research Center, Medical College of Varna, Medical University of Varna, Bulgaria, antoaneta_cvetkova@abv.bg

${ }^{4}$ Medical University of Plovdiv, Faculty of Pharmacy, Bulgaria, andreevska@abv.bg

${ }^{5}$ Medical University of Plovdiv, Faculty of Pharmacy, Bulgaria, grekovadaniela@gmail.com

${ }^{6}$ Medical University of Sofia,Faculty of Pharmacy, Bulgaria, petkovav1972@yahoo.com
} 
In the field of mental health, non-adherence to the therapy is a serious problem as the effectiveness of antidepressants decreases significantly when patients fail to follow their assigned treatment (Vergouwen et al., 2003). Non-adherence of the assigned therapy is considered to be the main cause in many cases for turning depression in a chronic condition. In recent years, adherence has been presented through two components - persistence (drug intake throughout the planned course of treatment) and compliance (Sawada et al., 2009, Dra. Et al., 2016). The main factors influencing the adherence to therapy are health system factors, social / economic factors, patient-related factors, therapy-related factors and factors related to the condition of the patient, and a number of other factors.

There are several types of non-adherence to the therapy:

- Primary non-adherence - the patient does not perform the assignment or misses a visit to the doctor.

- Secondarily - the patient does not perform the treatment in the prescribed manner.

- Conscious (intentional, deliberate) - the patient rejects the diagnosis or treatment. In this type of nonadherence, the communication with the patient is of utmost importance in order to understand his attitude towards his or her health problems.

- Unintentionality (unknowingly) may be due to a number of factors, for example, the patient would have taken the medicine, but the doses are over, they have forgotten to take it during a break, and so on. This species is most dependent on various demographic, social and clinical influences.

According to the literature, the following groups of factors determine the adherence of patients with depression:

1. Drug-related factors.

Complexity of the intake (number, type, dosage, etc.)

-The higher the number of doses, the less likely the medication is taken correctly.

The higher the dosage, the less likely the doses are to be taken by the patient (Petkova et al.,2015).

2. Accessible language of the patient's materials.

The leaflets of medicines, information materials, brochures and other materials are quite often misunderstood by the patient, given a number of factors such as age, literacy, and education that negatively affect the adherence to therapy (Horne et al., 1999).

3. Quality communication between doctor-patient-pharmacist.

The roles of the doctor and pharmacist are complementary, and it has been established that the expertise of pharmacists when channeled through a co-operative relationship with doctors has a positive impact on patient outcomes (Muller et al., 2006).

Good communication between the doctor, the patient and the pharmacist, combined with a flexible, non-judgmental and pragmatic attitude, can lead to improved results in terms of compliance with therapy and prognosis. Medical professionals should always allow the patient to express their concerns about the treatment and provide a balanced explanation of the benefit / risk ratio of medication as the remaining unresolved concerns of prolonged use of drugs are one of the main reasons for discontinuing adequate therapy.

\section{Data and methodology:}

The aim of the study is to analyze the degree of adherence to therapy in patients with a diagnosed depressive condition.

In order to achieve this goal, a survey was conducted among 120 patients, clients of pharmacies in the city of Varna with diagnosed depression when taking their prescription drugs. Determination of patient's compliance was done using the Morrisky test, and in parallel with that a survey was conducted giving information on gender, age, degree of education, and the number of medications taken. The Morriski's test was a short questionnaire giving a clear understanding of the degree of adherence to one's therapy. Used as a self-assessment method of compliance, it is the fastest questionnaire for identifying and evaluating the extent of adherence to therapy (Lavsa et al., 2011). It is composed of four questions with an open answer "Yes" and "No". Each answer "No" gets 1 point and the possible sum is from 0 to 4 . Patients with a higher score are assessed to show better adherence 
to therapy, and those with a lower score show an increased risk of non-adherence. Table 1 shows the score scale for assessing the degree of adherence to therapy.

\begin{tabular}{|l|l|}
\hline \multicolumn{2}{|l|}{ Table 1: Evaluation scale for adherence to therapy with the Morriski test. } \\
\hline Results & Degree of adherence to therapy \\
\hline $0-1$ points & Low degree of adherence \\
\hline $2-3$ points & Moderate degree adherence \\
\hline 4 points & High degree of adherence \\
\hline Source: Authors
\end{tabular}

\section{Results and Discussion:}

A survey of patients suffering from depressive disorder was conducted during visits to pharmacies in the city of Varna for medicines for the treatment of depressive conditions. 140 patients were interviewed, 120 of them filled out valid questionnaires. The remaining 20 patients had incomplete data and were excluded from the analysis.

The degree of adherence to therapy for the entire population was studied, while a number of other factors influencing the adherence to therapy were also considered, such as: gender, age, level of education, number of antidepressants and other medications.

The results of the surveys indicate that the share of women surveyed are with predominantly depressive disorders $(58,33 \%)$, while for men it was $41,67 \%$, which is also confirmed by date from the literature. Literary evidence suggests that women are twice as likely to be depressed than men. Some scientists point out social factors as the cause of higher levels of depressive disorder in women (Schäfer et al., 2012). Other literary sources indicate that $3 / 4$ of the depressed patients are females (Kuehner et al., 2003). The distribution of patients by sex is presented in Figure 1.

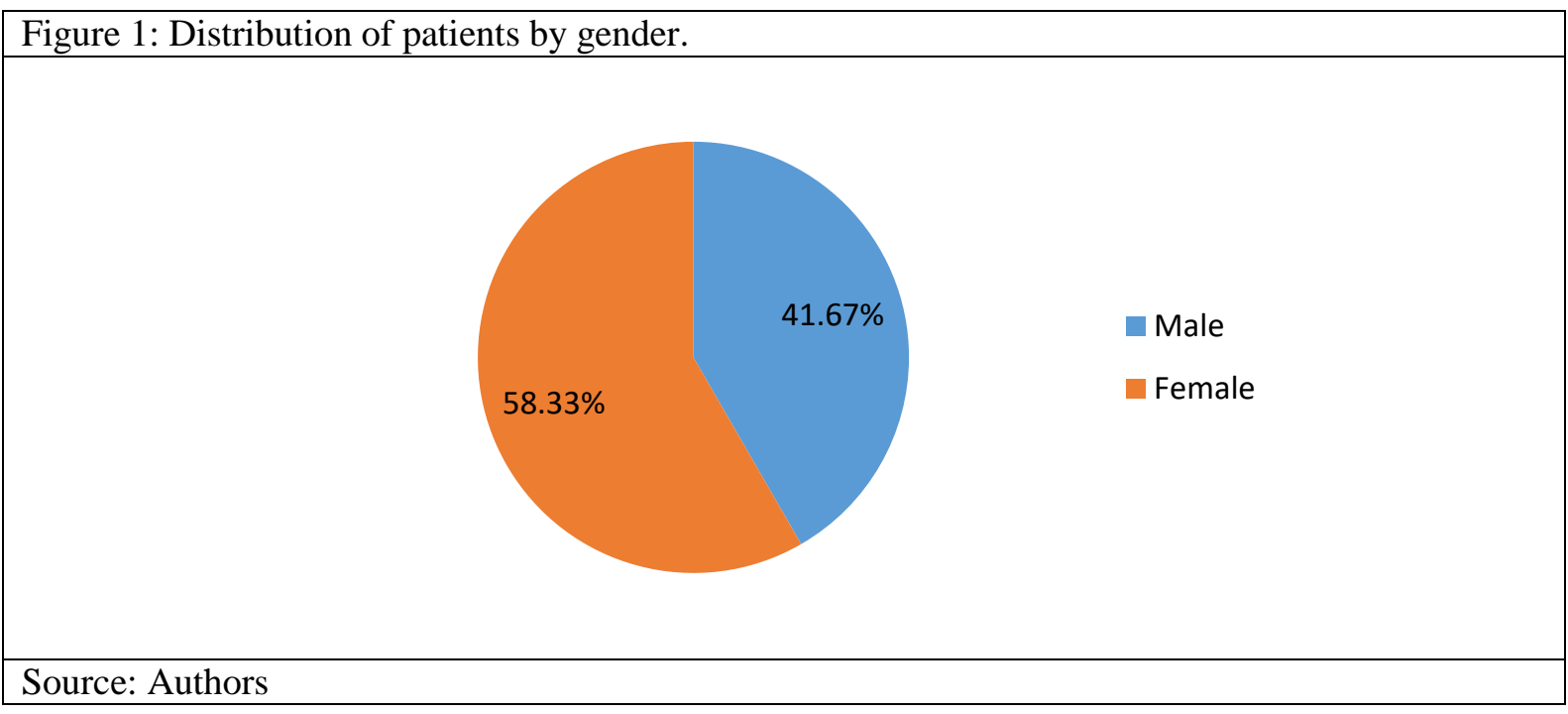

The average age of the patients who were interviewed is approximately 53years. According to literary sources, the consumption of antidepressants is higher in adult patients (Fourrier et al., 1996).

In our study, the distribution of participants with depressive symptoms at 40-65 years of age occupies the largest share of 56\%, followed by the $25-40$ age group, which occupies $25 \%$ and the lowest share is the group over 65 years. (19\%). According to other studies, depressive disorder is most commonly seen in the mid-to late 30s (Schäfer et al.,2012). As a result of the data obtained, we can observe that with increasing age depression is more pronounced. The distribution of patients by age is presented in Figure 2.

The results of the survey show that the majority of respondents have secondary education. These patients occupy the largest share of $57 \%$, followed by those with college education - $23 \%$. Patients with higher education account for a relatively small share of total patients $-12 \%$. Extremely low are those with primary and secondary education. The degree of completion of education is an essential factor for the degree of adherence to therapy. Literary data indicate that primary and secondary 
education have a greater difficulty in following therapy (Akpa et al., 2005). The distribution of patients versus the level of education is presented in Figure 3.

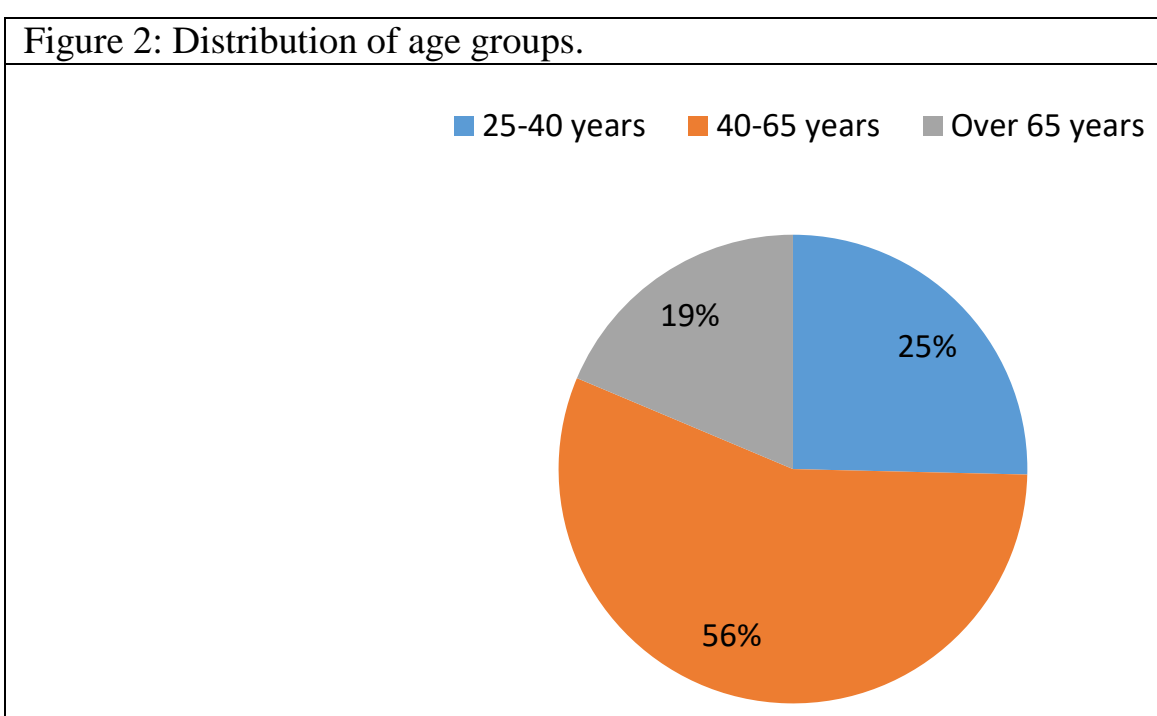

Source: Authors

Figure 3: Distribution of patients by level of education.

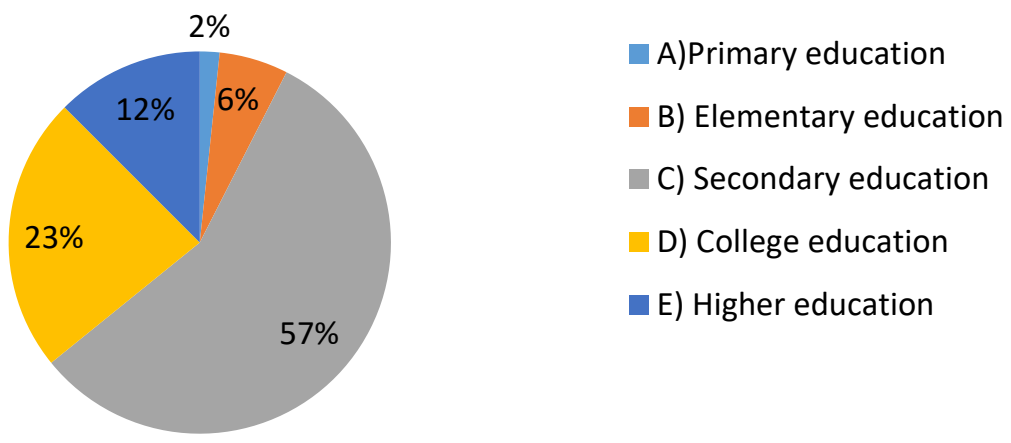

Source: Authors

Figure 4: Distribution of daily antidepressants intake.

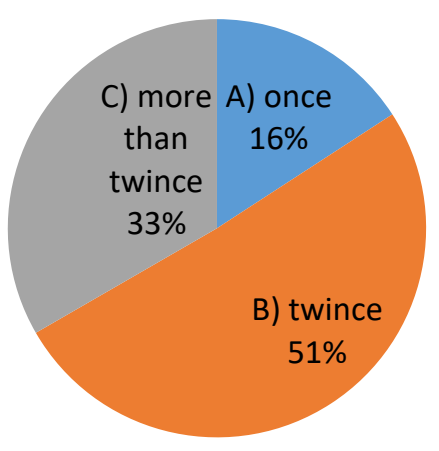

Source: Authors 
Patients were asked how many times a day they need to take antidepressants. A relatively large proportion of patients (51\%) take antidepressants twice a day. With a relatively high percentage $(33 \%)$, there are patients who have responded that they need to take antidepressants more than twice a day. Only $16 \%$ of patients surveyed responded once a day. Literary evidence suggests that the greater and more complicated the intake of drugs is, the less likely it is for patients to adhere to the intended therapy. Complex drug intake is one of the factors for improper treatment. The results for the distribution of daily antidepressant intakes are presented in Figure 4.

The answer to our question, "How many times a day are you taking other medicines than your appointed antidepressants?" $50 \%$ of the patients responded that they had to take them twice a day. A relatively small proportion of 33\% responded more than once, and only 17\% responded once a day. The results are presented in Figure 5.

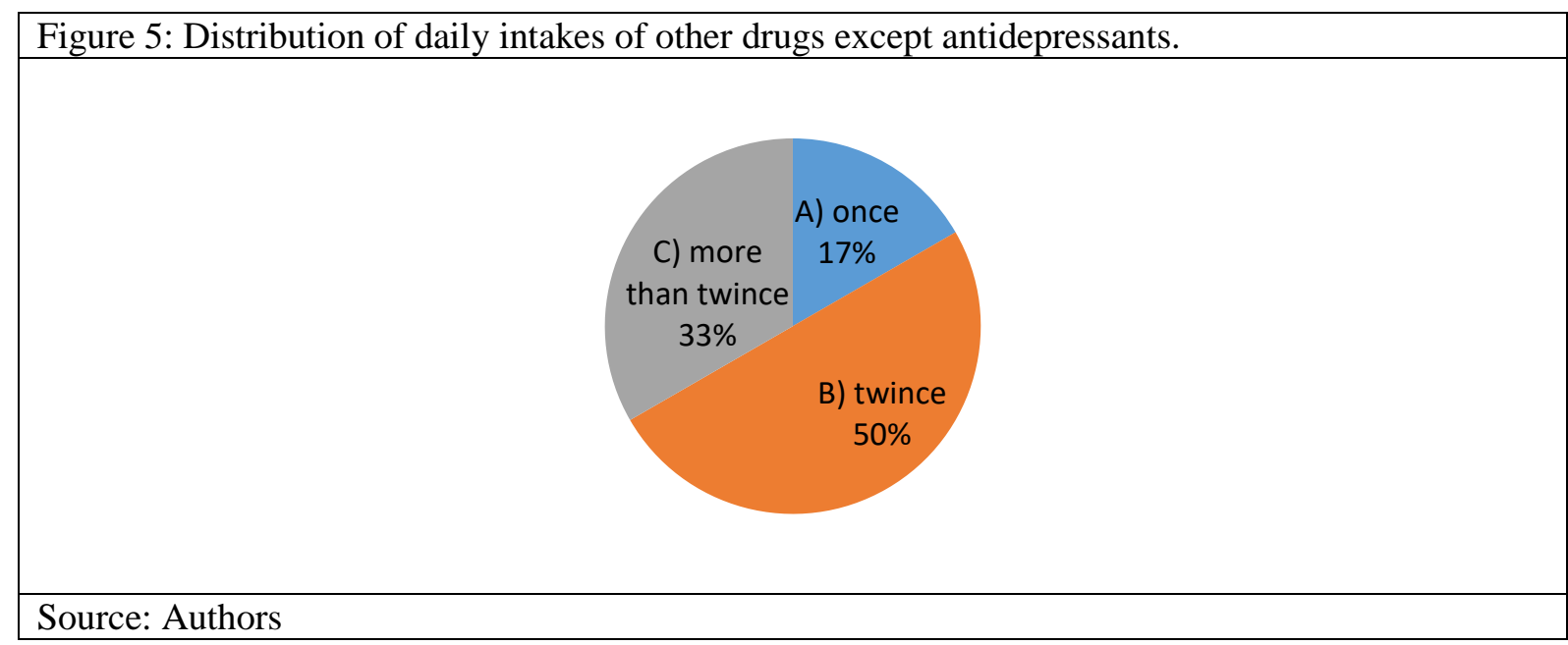

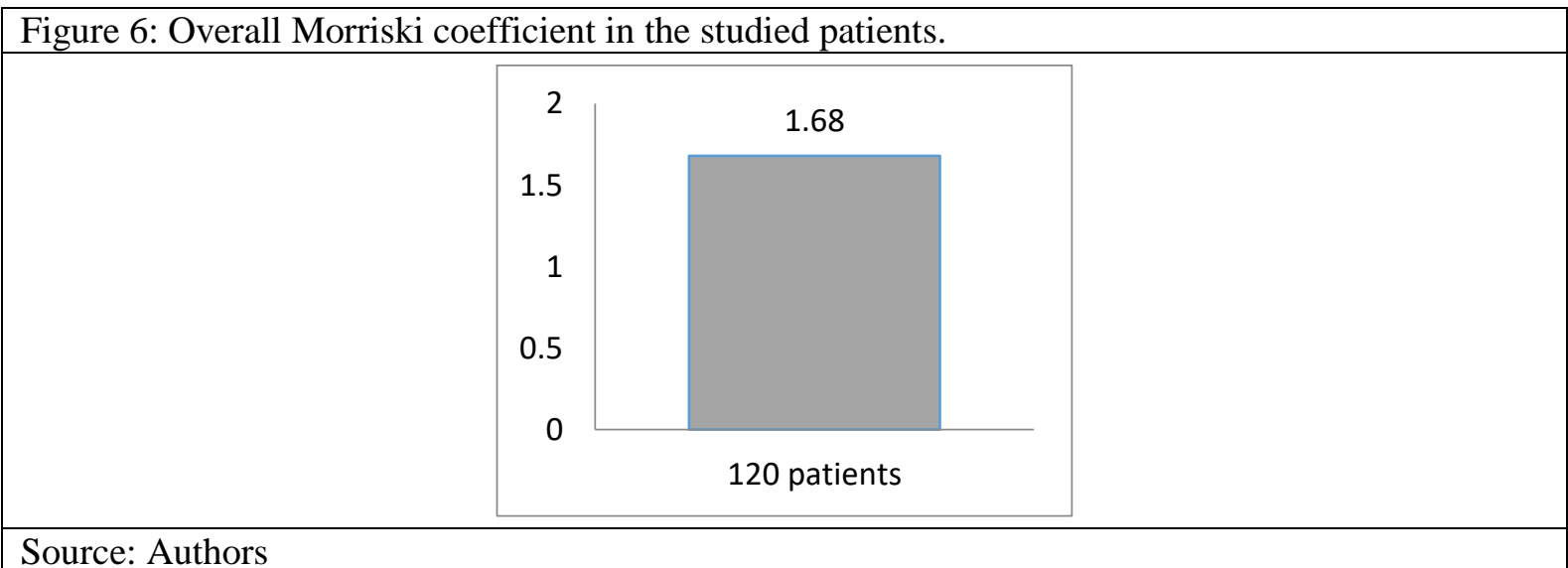

Source: Authors

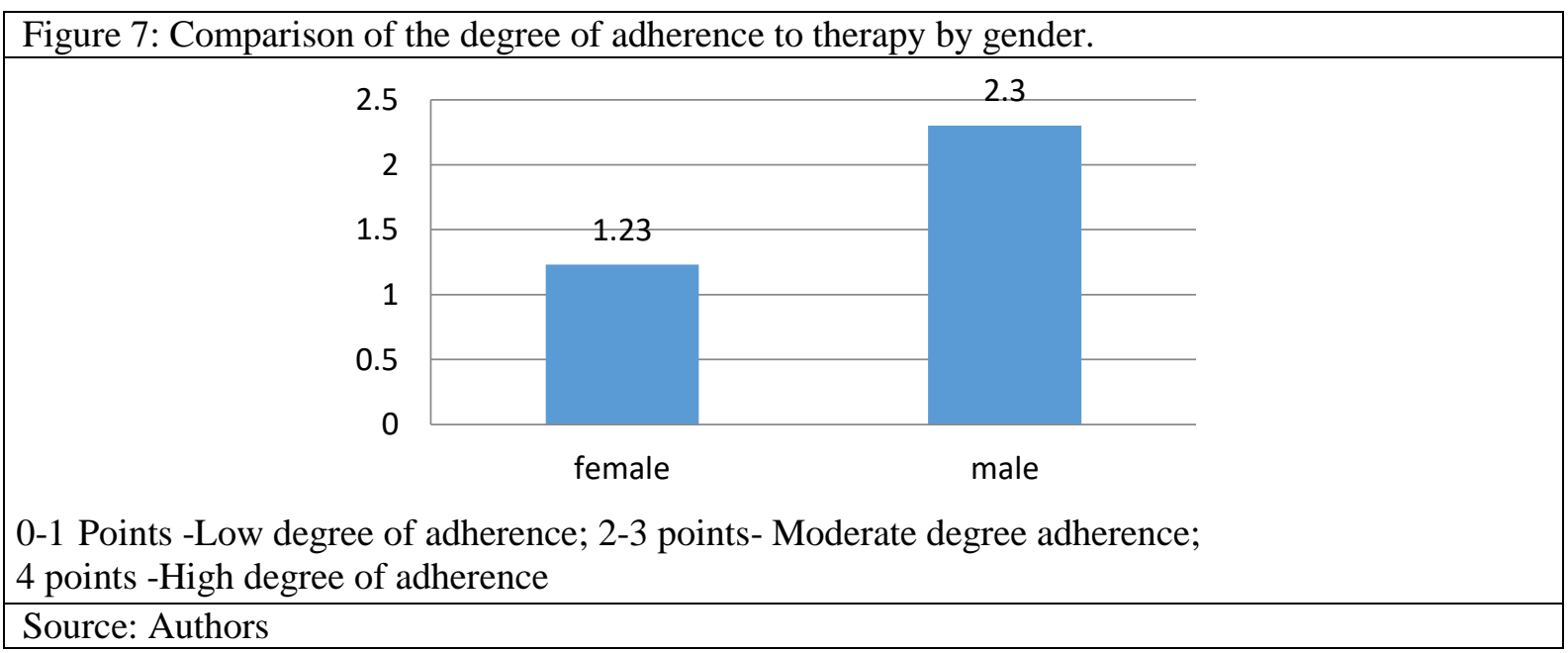


Results of the Morrisky test for adherence to the prescribed therapy of patients with a diagnosed depressive disorder prior to pharmaceutical care. The Morrisky's test was applied to these 120 patients and the established adherence to therapy was valued unsatisfactory. The total coefficient we received for 120 patients was 1.68. By comparing it with the Morrisky's adherence test reference, a 1.68 means a low degree of adherence to therapy. The results are presented graphically in Figure 6.

Subsequently, the patients were divided by gender, as follows: 70 women and 50 men.

The study found that men had a much better degree of adherence to therapy than women. The results of the Morrisky coefficient are plotted in Figure 7.

By analyzing the results obtained, we see that women have a holding coefficient of 1.23 , comparing them with the reference values, they fall into the low-adherence zone. Unlike women, men show better adherence to therapy with a factor of 2.3. By comparing the ratio of men to the reference values, we can say that men have a moderate degree of adherence to therapy.

Adherence to antidepressant therapy is a major problem in patients with depressive disorders. Early discontinuation of antidepressant therapy is statistically associated with $77 \%$ of cases of recurrence of depression. Literary data indicate that the socio-economic status, the stigma of antidepressants, adverse drug reactions, poor therapeutic approach, and complicated intake are factors of poor adherence to therapy. The statistics on non-compliance are extremely worrying, despite the enormous efforts made in recent years to find a solution to the problem, with between $20 \%$ and $80 \%$ of patients taking their medication regularly showing unsatisfactory therapy. Non-treatment is one of the greatest challenges for patients and health professionals. A number of psychological approaches and professional health support can be useful for better patient compliance with therapy.

\section{Conclusion:}

Improper adherence to therapy is a major problem for successful treatment and leads to a worsening of the condition of patients with depression while also increasing the cost of health care. A number of measures are needed, such as patient education, quality communication between physicians, patients, and pharmacists, socio-economic measures that change patient's beliefs and perceptions of treatment, and improved adherence to treatment and quality of life for depressed patients.

\section{References:}

Dra. Maria-Jose Martin-Vazquez†, Adherence to antidepressants: A review of the literature, Neuropsychiatry (London), 2016; 6(5): 236-241.

Elena Petkova, Dimitar Popov, Complications and adherence to bronchial asthma therapy, In Spiro, 2015, 5 (33): 21.

Fourrier A, Letenneur L, Dartigues J, Decamps A, Bégaud B. Consommation médicamenteuse chez le sujet âgé vivant à domicile et en institution à partir de la cohorte Paquid : importance de la polymédication et utilisation des psychotropes. Rev Gériatrie ,1996; (21) : 473-82.

Horne R, Weinman J. Patients' beliefs about prescribed medicines and their role in adherence to treatment in chronic physical illness. J Psychosom Res 1999; 47:555-67.

Kuehner Christine, Gender differences in unipolar depression: an update of epidemiologicalfindings and possible explanations, Acta Psychiatrica Scandinavica, 2003,108 (3):161-248.

Lavsa S. M., Holzworth A., Ansani N. T. Selection of a validated scale for measuring medication adherence. Journal of the American Pharmacists Association. 2011; 51(1):90-94.

Muller BA, McDanel DL.Enhancing quality and safety through physician- pharmacist collaboration. American Journal of Health-System Pharmacy, 2006; 63:996-7.

Sawada N, Uchida H, Suzuki T, et al. Persistence and compliance to antidepressant treatment in patients with depression: a chart review. BMC. Psychiatry, 2009, 16(1): 9-38.

Schäfer I., Heike Hansen, Gerhard Schön, Susanne Höfels, Attila Altiner, Anne Dahlhaus, Jochen Gensichen, The influence of age, gender and socio-economic status on multimorbidity patterns in primary care,first results from the multicare cohort study, BMC Health Services Research 2012, 12:89.

Schäfer I., Heike Hansen, Gerhard Schön, Susanne Höfels, Attila Altiner, Anne Dahlhaus, Jochen Gensichen, The influence of age, gender and socio-economic status on multimorbidity patterns in primary care,first results from the multicare cohort study, BMC Health Services Research 2012, 12:90.

Vergouwen A, Bakker A, Koerselman F. Adherence to medication for chronic psychiatric diseases: determining the optimum frequency and form of administration. Am. J. Drug. Deliv 2003,1(4), 267-273.

World Federation for Mental Health, Depression: A global crisis, World Mental Health Day, October 10, 2012.

Akpa MR, Agomuoh DI, Odia OJ. Drug compliance among hypertensive patients in Port Harcourt, Nigeria. Niger J Med. 2005; 14:55-7. 